\title{
Bell Inequality from Holographic Gravity
}

\author{
Sichun Sun ${ }^{1}$ and Yun-Long Zhang ${ }^{2, \star}$ \\ ${ }^{1}$ Center for Theoretical Sciences, National Taiwan University, Taipei 10617, Taiwan \\ ${ }^{2}$ Asia Pacific Center for Theoretical Physics, Pohang 790-784, Korea
}

\begin{abstract}
We study a holographic model of an EPR pair at the boundary of bulk gravity, and use Bell inequality as a sharp test of entanglement. By revealing how Bell inequality is violated by gravity in the bulk, our study sheds light on the entanglement of the original ER=EPR conjecture.
\end{abstract}

\section{Introduction}

This proceeding is mainly base on [1], where the Bell inequality from holographic gravity is constructed. Correlations in local classical theories are bounded by the Bell inequality, however, it can be violated by the presence of the non-local entanglement in quantum mechanics [2]. The violation of Bell inequality in the entangled EinsteinPodolsky-Rosen (EPR) pair [3] indicates that two particles have an "instant interaction", in contrast to the hidden variable theories that preserve strict locality [2-6]. In addition to the Bell's tests in laboratories, there are also discussions of detections in cosmological scales, to confirm that whether the inflated primordial fluctuations are quantum mechanical [7-9]. Recently, the ER=EPR conjecture is proposed by Maldacena and Susskind [10, 11]. It stated that the quantum entanglement of the EPR pair can be attributed to the non-traversable Einstein-Rosen (ER) bridge that connects the pair [12]. This conjecture was proposed to resolve the black hole information paradox without resorting to a firewall [13] surround the black hole horizon.

In this work, instead of working with the original $\mathrm{ER}=\mathrm{EPR}$ setup, we employ a concrete holographic model of the EPR pair proposed by Jensen and Karch in Ref [14] based on the Anti-de Sitter/Conformal Field Theory(AdS/CFT) correspondence [15] and the analytical solution in [16]. The two particles of the boundary EPR pair are connected by a string in the AdS background of the bulk, with an effective ER bridge on the string worldsheet. Therefore, this is a holographic realization of ER=EPR conjecture.

We demonstrate that the Bell inequality violated by the EPR pair living at the boundary can also be violated by the gravitational theory with an ER bridge living in the bulk. Although the holographic setting is different from the original $\mathrm{ER}=\mathrm{EPR}$ conjecture in which both ER and EPR live

${ }^{\star}$ Corresponding Author and Speaker: yunlong.zhang@apctp.org. Proceeding for the 13th International Conference on Gravitation, Astrophysics, and Cosmology the 15th Italian-Korean Symposium on Relativistic Astrophysics: A Joint Meeting. Parallel Talk on July 6, 2017, Seoul, Korea. in the same spacetime dimensions, our study does shed light on how entanglement can be captured by a classical theory.

\section{Holographic EPR Pair}

In this section, we review the holographic model of the EPR pair in Ref. [14]. The model proposed that an entangled color singlet quark anti-quark $(q-\bar{q})$ pair in $\mathcal{N}=4$ supersymmetric Young-Mills theory (SYM) can be described by an open string with both of its endpoints attached to the boundary of $\mathrm{AdS}_{5}$. There is no confinement in this theory and the pair can separate arbitrarily far away from each other. Relevant studies of this model can also be found in [17-23].

There are numerical solutions of the string shapes with different boundary behaviors [24-26]. We will work with the analytic solution for an accelerating string treated in the probe limit at the AdS background [16]. In the analytic solution the open string is accelerated on the Poincáre patch of the $\mathrm{AdS}_{5}$ with radius $L$,

$$
\mathrm{d} s^{2}=\frac{L^{2}}{w^{2}}\left[-\mathrm{d} t^{2}+\mathrm{d} w^{2}+\left(\mathrm{d} x^{2}+\mathrm{d} y^{2}+\mathrm{d} z^{2}\right)\right]
$$

The string solution in the bulk gravity is given by

$$
z^{2}=t^{2}+b^{2}-w^{2}
$$

The quark and anti-quark live on the AdS boundary $w=0$. They are accelerating along the $\pm z$ direction, respectively, with the solution $z= \pm \sqrt{t^{2}+b^{2}}$. Therefore, the two entangled particles are out of causal contact with each other the whole time.

String fluctuations. - To consider the string fluctuations, we transform the solution to the co-moving spacetime $(\tilde{\tau}, \tilde{r}, x, y, \tilde{z})$ of the accelerating quarks via

$$
\begin{aligned}
|z| & =b \sqrt{1-\tilde{r}} e^{\tilde{z}} \cosh \tilde{\tau}, \\
t & =b \sqrt{1-\tilde{r}} e^{\tilde{z}} \sinh \tilde{\tau},
\end{aligned}
$$




$$
w=b \sqrt{\tilde{r}} e^{\tilde{z}}, \quad x=b \tilde{x}, \quad y=b \tilde{y} .
$$

These two frames, which cover the regions $z \geq 0$ and $z \leq 0$ separately, are accelerating frames with a constant acceleration $a=1 / b$ along opposite directions of $z$. And (3) only maps the upper part of the string $(0<w<b)$ into the proper frames of the accelerating quarks with $0<\tilde{r}<1$. Plug this transformation (3) in the string solution (2), the string configuration becomes $\tilde{z}=0$ for both frames of the quark and anti-quark. Under this transformation (3), the metric (1) becomes

$$
\mathrm{d} s^{2}=\frac{L^{2}}{\tilde{r}}\left[-f(\tilde{r}) \mathrm{d} \tilde{\tau}^{2}+\frac{1}{4 \tilde{r}} \frac{\mathrm{d} \tilde{r}^{2}}{f(\tilde{r})}+e^{-2 \tilde{z}}\left(\mathrm{~d} \tilde{x}^{2}+\mathrm{d} \tilde{y}^{2}\right)+\mathrm{d} \tilde{z}^{2}\right],
$$

where $f(\tilde{r})=1-\tilde{r}$. Furthermore, with respect to the time $\tau=b \tilde{\tau}$, the Hawking temperature $T_{H}=\frac{1}{2 \pi b}$ matches with the Unruh temperature $T_{a}=\frac{a}{2 \pi}[16,23]$ and we have set the reduced Planck constant $\hbar$ and Boltzmann constant $k_{B}$ to be unit. Therefore, there is an event horizon at $\tilde{r}=1$ associated with the quark and another event horizon also at $\tilde{r}=1$ associated with the anti-quark. As shown in Fig. 1, the two horizons are connected by part of the string which can be seen as ER bridge. Hence it is suggested to be a holographic realization of the ER=EPR conjecture [10, $11]$.

The measurement of the quarks' states will introduce fluctuations to the world lines of quarks which set the boundary conditions for the worldsheet of the string fluctuations. Let $(\tilde{\tau}, \tilde{r})$ be the new worldsheet coordinates in the current frame, then the string fluctuation is $X^{\mu}(\tilde{\tau}, \tilde{r})=$ $\left(\tilde{\tau}, \tilde{r}, \tilde{q}_{i}(\tilde{\tau}, \tilde{r})\right)$, with $i=(\tilde{x}, \tilde{y}, \tilde{z})$. When $\tilde{q}_{i} \ll 1$, the NambuGoto action of string with tension $T_{s}$ becomes

$$
S \simeq-T_{s} L^{2} \int \frac{d \tilde{\tau} d \tilde{r}}{2 \tilde{r}^{3 / 2}}\left\{1+\left[2 \tilde{r} f(\tilde{r}) \tilde{q}_{i}^{\prime} \tilde{q}_{j}^{\prime}-\frac{1}{2 f(\tilde{r})} \dot{\tilde{q}}_{i} \dot{\tilde{q}}_{j}\right] h^{i j}\right\},
$$

where $\tilde{q}_{i}^{\prime} \equiv \frac{\partial \tilde{q}_{i}}{\partial \tilde{r}}, \dot{\tilde{q}}_{i} \equiv \frac{\partial \tilde{q}_{i}}{\partial \tilde{\tau}}$ and $h^{i j}=\operatorname{diag}\left[1, e^{2 \tilde{z}}, e^{2 \tilde{z}}\right]$. The equations of motion for the fluctuations on the string are

$$
\partial_{\tilde{r}}\left(\frac{2 f \tilde{q}_{i}^{\prime}}{\tilde{r}^{1 / 2}}\right)-\partial_{\tilde{\tau}}\left(\frac{\dot{\tilde{q}}_{i}}{2 f \tilde{r}^{3 / 2}}\right)=0
$$

Focusing on the transverse fluctuations $i=\tilde{x}, \tilde{y}$,

$$
\tilde{q}_{i}(\tilde{\tau}, \tilde{r})=\int \frac{d \omega}{2 \pi} e^{-i \omega \tilde{\tau}} \tilde{q}_{i}(\omega) Y_{\omega}(\tilde{r}),
$$

where $\tilde{q}_{i}(\omega)$ is the Fourier transform of fluctuation on the boundary. With these we can study the Green's function holographically.

\section{Constructing Bell's inequality}

The retarded Green's function of the quark under effective random force $\mathcal{F}^{i}(\tau)$ can be defined as $\mathbf{i} G_{\mathcal{R}}^{i j}(\tau)=$ $\theta(\tau)\left\langle\left[\mathcal{F}^{i}(\tau), \mathcal{F}^{j}(0)\right]\right\rangle[16]$. In the AdS/CFT correspondence, $\mathcal{F}^{i}(\tau)$ is the operator conjugate to the fluctuations $q_{i}(\tau)$, where the dimensionful quantities are $\tau=b \tilde{\tau}, q_{i}=$

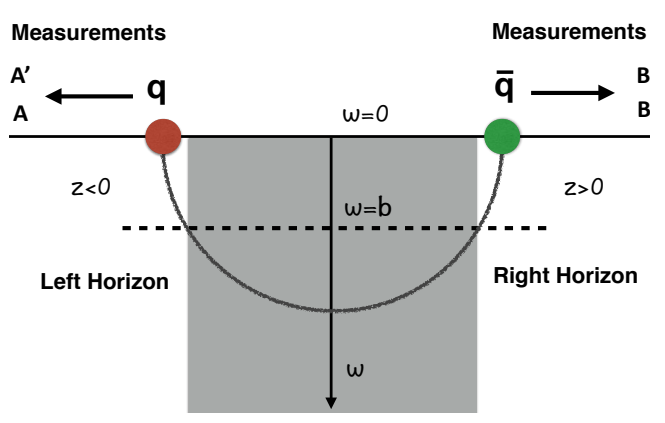

Figure 1. Schematic diagram for the quark $(q)$-antiquark $(\bar{q})$ EPR pair in holography at a fixed time $t>0$. The trajectory of left(right) worldsheet horizon is on $w=b$, which depicts the intersection of the string with the world volume horizons seen by $q(\bar{q})$ in its co-moving frame. The world volume horizons are the two vertical boundary of the gray region. The Bell inequality test is performed by the measurements at Alice and Bob's locations.

$b \tilde{q}_{i}$. In the low frequency limit $\omega \rightarrow 0$, it can be obtained analytically as

$$
\begin{aligned}
G_{\mathcal{R}}^{i j}(\omega) & =-\left.\frac{2 T_{s} L^{2}}{b^{2} \tilde{r}^{1 / 2}} f(\tilde{r}) Y_{-\omega}(\tilde{r}) \partial_{\tilde{r}} Y_{\omega}(\tilde{r}) \delta^{i j}\right|_{\tilde{r} \rightarrow 0} \\
& =-\frac{a^{2} \sqrt{\lambda}}{2 \pi} \mathbf{i} \omega \delta^{i j}+O\left(\omega^{2}\right),
\end{aligned}
$$

and we have used the fact that $T_{s} L^{2}=\frac{\sqrt{\lambda}}{2 \pi}$.

What we need for the Bell's test is the contour time ordered Schwinger-Keldysh (SK) Green's function,

$$
\mathbf{i} G_{A B}^{i j}(\tau, x)=\left\langle\mathcal{F}_{A}^{i}(\tau, x) \mathcal{F}_{B}^{j}(0)\right\rangle,
$$

where $\mathcal{F}_{A}^{i}$ and $\mathcal{F}_{B}^{j}$ are separately defined on the causally disconnected left and right wedges of the Penrose diagram, corresponding to the boundaries of different patches of the AdS space. This off-diagonal SK propagator is examined in the Supplemental Material and found to be related to the holographic retarded Green's function

$$
G_{A B}^{i j}(\omega)=\frac{2 \mathbf{i} e^{-\omega /\left(2 T_{a}\right)}}{1-e^{-\omega / T_{a}}} \operatorname{Im} G_{\mathcal{R}}^{i j}(\omega),
$$

similar to what was found in Refs. [27-29] but with different settings.

For fluctuations coming from two causally separated quarks of an EPR pair along $x, y$ directions, and in the low frequency limit $\omega \rightarrow 0$,

$$
\mathbf{i} G_{A B}^{x x}=\mathbf{i} G_{A B}^{y y}=\frac{\sqrt{\lambda} a^{3}}{2 \pi^{2}}, \quad \mathbf{i} G_{A B}^{x y}=\mathbf{i} G_{A B}^{y x}=0,
$$

which indicates that the spatial correlator $G_{A B}^{i j} \propto \delta^{i j}$. The $\sqrt{\lambda}$ factor is consistent with the observation that the entanglement entropy of the entangled pair is of order $\sqrt{\lambda}$ [14]. It is also interesting that this SK correlator does not vanish when the quarks are separated at long distance. This is consistent with the non-local nature of entanglement.

To study the correlators, we normalize the operators such that only the dependence on the spin wave function remains:

$$
A_{\mathcal{F}}=\left(\cos \theta_{A} \mathcal{F}_{A}^{x}+\sin \theta_{A} \mathcal{F}_{A}^{y}\right) /\left\langle\mathcal{F}_{A}^{x} \mathcal{F}_{B}^{x}\right\rangle^{1 / 2},
$$




$$
B_{\mathcal{F}}=\left(\cos \theta_{B} \mathcal{F}_{B}^{x}+\sin \theta_{B} \mathcal{F}_{B}^{y}\right) /\left\langle\mathcal{F}_{A}^{x} \mathcal{F}_{B}^{x}\right\rangle^{1 / 2},
$$

The mixed measurements for correlators in CHSH correlation formulation become

$$
\left\langle A_{\mathcal{F}} B_{\mathcal{F}}\right\rangle=\cos \left(\theta_{A}-\theta_{B}\right) \equiv \cos \theta_{A B}
$$

Together with the similar normalization of the operators $A_{\mathcal{F}}^{\prime}$ and $B_{\mathcal{F}}^{\prime}$, the $\mathrm{CHSH}$ correlation formulations becomes

$$
\begin{aligned}
\left\langle C_{\mathcal{F}}\right\rangle & =\left\langle A_{\mathcal{F}} B_{\mathcal{F}}\right\rangle+\left\langle A_{\mathcal{F}} B_{\mathcal{F}}^{\prime}\right\rangle+\left\langle A_{\mathcal{F}}^{\prime} B_{\mathcal{F}}\right\rangle-\left\langle A_{\mathcal{F}}^{\prime} B_{\mathcal{F}}^{\prime}\right\rangle \\
& =\cos \theta_{A B}+\cos \theta_{A B^{\prime}}+\cos \theta_{A^{\prime} B}-\cos \theta_{A^{\prime} B^{\prime}} .
\end{aligned}
$$

For example, when $\theta_{A B}=\theta_{A B^{\prime}}=\theta_{A^{\prime} B}=\pi / 4$, and $\theta_{A^{\prime} B^{\prime}}=$ $3 \pi / 4$, we can reach the maximum value $2 \sqrt{2}$.

In this derivation, we see the bulk string fluctuations, which come from classical gravity, reproduce the quantum entanglement of an EPR pair on the boundary. The equation of motion of the classical string, Eq.(6), has no coupling between $\tilde{q}_{x}$ and $\tilde{q}_{y}$ such that Eq.(11) follows. This can be obtained as long as the string does not experience a force to propagate the fluctuation in the $x$-direction to the $y$-direction which breaks parity in general. It seems once these two conditions are satisfied, it does not matter whether there is an ER bridge in the bulk. Hence it is conceivable Bell inequality can still be violated in a holographic model where the EPR pair does not accelerate, similar to how holographic entanglement entropy is computed in a static system $[30,31]$.

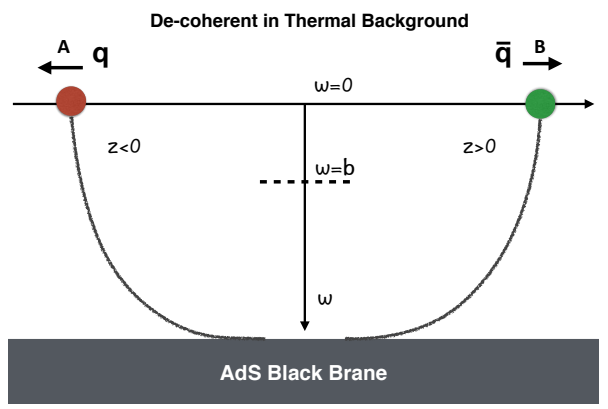

Figure 2. Schematic diagram for the decoherence of quark $(q)$ antiquark $(\bar{q})$ EPR pair in holography at late time. The string breaks after it fell into the black brane. This process correspondends to the decoherence of the quark and anti quak pairs induced by thermal background.

\section{Time Evolution and Discussion}

It is interesting to consider the decoherence of the EPR pair in the environment. If the environmental effect can be described by thermal fluctuations, then we can add a black brane to the bulk of our model. When the distance of the EPR pair increases with time, the ER bridge also approaches the black brane horizon and then enters the horizon [32]. We expect the ER bridge breaks after it enters the horizon which might shed light on the decoherence process in the boundary field theory. As shown in Fig. 2,
We would also like to comment on the finite temperature effects on the accelerating string solution in $\mathrm{AdS}_{5}$ black brane spacetime. Although there is no analytical solution of the string expansion, qualitatively the string will touch the horizon of the black hole at late time. There is a semi-quantitative discussion in the small temperature limit [32], that the Hawking temperature of the black hole is much smaller that the Unruh temperature of the accelerating particle $T_{H} \ll T_{a}$. An external electric field is imposed to accelerate the quark-antiquark pair from $t=0$ to $t=\infty$. It is expected at late time energy is flowing into the system from the top of the string to the bottom part, and then being absorbed by the black brane. As time increases, the acceleration slows down and the string trajectory starts to evolve towards the trailing string solution. This corresponds to that the Einstein-Rose bridge is broken up by the horizon of AdS black brane. And for the static string solution in the AdS black brane where two straight strings is hanging down to the horizon, there is no entanglement in the dual state [24]. Other approach to describe quantum operations in holography can also be found in [31].

For future work, it is interesting to consider the back reaction by the measurements and see whether the ER bridge is broken due to the energy injected by measurements. This might provide an opportunity to study the "wave function collapse" typically used to describe how measurements change the states. For our case in pure AdS, if we consider the back reaction after the measurements, the ER bridge might be broken due to the energy injected by measurements, similar to the case with black brane. This is aligned with the fact that the wave function of the EPR pair will collapse after the measurements. Since the string is in probe limit, it is not explicit that the bridge will break due to the string perturbations.

The ER=EPR conjecture is proposed to resolve the black hole information paradox without introducing a firewall in the black hole. The conjecture implies that entanglement of the EPR pair, which is thought to be a quantum mechanical effect, can be captured by classical gravity through the ER bridge. Using Bell inequality as a sharp test of entanglement, we study a holographic model with an EPR pair at the boundary and an ER bridge in the bulk. By revealing how Bell inequality is violated by classical gravity in the bulk, our study sheds light on the possible conditions needed for the entanglement of the original $\mathrm{ER}=\mathrm{EPR}$.

Acknowledgements - Y. L. Zhang thanks the supported by APCTP for the conference. We thank D. Berenstein, J. W. Chen, A. Karch, Y. Neiman for helpful communication.

\section{References}

[1] J. W. Chen, S. Sun and Y. L. Zhang, "Holographic Bell Inequality," arXiv:1612.09513 [hep-th].

[2] J. S. Bell, "On the Einstein-Podolsky-Rosen paradox," Physics 1, 195 (1964).

[3] A. Einstein, B. Podolsky and N. Rosen, "Can quantum mechanical description of physical reality be considered complete?," Phys. Rev. 47, 777 (1935). 
[4] J. F. Clauser, M.A. Horne, A. Shimony, R.A. Holt, "Proposed experiment to test local hidden-variable theories," Phys. Rev. Lett. 23, 880 (1969).

[5] B. S. Cirelson, "Quantum generalizations of Bell's inequality,” Lett. Math. Phys. 4, 93 (1980).

[6] J. B. Hartle, "Space-time quantum mechanics and the quantum mechanics of space-time," gr-qc/9304006.

[7] J. Maldacena, "A model with cosmological Bell inequalities," Fortsch. Phys. 64, 10 (2016) [arXiv:1508.01082 [hep-th]].

[8] S. Choudhury, S. Panda and R. Singh, "Bell violation in the Sky," Eur. Phys. J. C 77, no. 2, 60 (2017) [arXiv:1607.00237 [hep-th]].

[9] J. W. Chen, S. H. Dai, D. Maity, S. Sun and Y. L. Zhang, "Towards Searching for Entangled Photons in the CMB Sky," arXiv:1701.03437 [quant-ph].

[10] J. Maldacena and L. Susskind, "Cool horizons for entangled black holes,” Fortsch. Phys. 61, 781 (2013) [arXiv:1306.0533 [hep-th]].

[11] L. Susskind, "Copenhagen vs Everett, Teleportation, and ER=EPR," Fortsch. Phys. 64, no. 6-7, 551 (2016) [arXiv:1604.02589 [hep-th]].

[12] A. Einstein and N. Rosen, "The Particle Problem in the General Theory of Relativity," Phys. Rev. 48, 73 (1935).

[13] A. Almheiri, D. Marolf, J. Polchinski and J. Sully, "Black Holes: Complementarity or Firewalls?," JHEP 1302, 062 (2013) [arXiv:1207.3123 [hep-th]].

[14] K. Jensen and A. Karch, "Holographic Dual of an Einstein-Podolsky-Rosen Pair has a Wormhole," Phys. Rev. Lett. 111, no. 21, 211602 (2013) [arXiv:1307.1132 [hep-th]].

[15] J. M. Maldacena, "The Large N limit of superconformal field theories and supergravity," [Adv. Theor. Math. Phys. 2, 231 (1998)] [hep-th/9711200].

[16] B. W. Xiao, "On the exact solution of the accelerating string in $\operatorname{AdS}(5)$ space," Phys. Lett. B 665, 173 (2008) [arXiv:0804.1343 [hep-th]].

[17] J. Sonner, "Holographic Schwinger Effect and the Geometry of Entanglement," Phys. Rev. Lett. 111, no. 21, 211603 (2013) [[arXiv:1307.6850 [hep-th]].

[18] K. Jensen, A. Karch and B. Robinson, "Holographic dual of a Hawking pair has a wormhole," Phys. Rev. D 90, no. 6, 064019 (2014) [arXiv:1405.2065 [hepth]].

[19] K. Jensen and J. Sonner, "Wormholes and entanglement in holography,” Int. J. Mod. Phys. D 23, no. 12,
1442003 (2014) [arXiv:1405.4817 [hep-th]].

[20] M. Chernicoff, A. Güijosa and J. F. Pedraza, "Holographic EPR Pairs, Wormholes and Radiation," JHEP 1310, 211 (2013) [arXiv:1308.3695 [hep-th]].

[21] A. Karch and S. Sun, "Matrix Flavor Brane and Dual Wilson Line,” Phys. Rev. D 89, no. 6, 066008 (2014) [arXiv:1312.2694 [hep-th]].

[22] T. Hirayama, P. W. Kao, S. Kawamoto and F. L. Lin, "Unruh effect and Holography," Nucl. Phys. B 844, 1 (2011) [arXiv:1001.1289 [hep-th]].

[23] E. Caceres, M. Chernicoff, A. Guijosa and J. F. Pedraza, "Quantum Fluctuations and the Unruh Effect in Strongly-Coupled Conformal Field Theories," JHEP 1006, 078 (2010) [arXiv:1003.5332 [hep-th]].

[24] C. P. Herzog, A. Karch, P. Kovtun, C. Kozcaz and L. G. Yaffe, "Energy loss of a heavy quark moving through $\mathrm{N}=4$ supersymmetric Yang-Mills plasma," JHEP 0607, 013 (2006) [hep-th/0605158].

[25] M. Chernicoff and A. Guijosa, "Acceleration, Energy Loss and Screening in Strongly-Coupled Gauge Theories," JHEP 0806, 005 (2008) [arXiv:0803.3070 [hep-th]].

[26] P. M. Chesler, K. Jensen and A. Karch, "Jets in strongly-coupled N = 4 super Yang-Mills theory," Phys. Rev. D 79, 025021 (2009) [arXiv:0804.3110 [hep-th]].

[27] D. T. Son and A. O. Starinets, "Minkowski-space correlators in AdS/CFT correspondence: Recipe and applications," JHEP 0209, 042 (2002). [arXiv:hepth/0205051].

[28] C. P. Herzog and D. T. Son, "Schwinger-Keldysh propagators from AdS/CFT correspondence," JHEP 0303, 046 (2003). [arXiv:hep-th/0212072].

[29] D. T. Son and A. O. Starinets, "Viscosity, Black Holes, and Quantum Field Theory," Ann. Rev. Nucl. Part. Sci. 57, 95 (2007) [arXiv:0704.0240 [hep-th]].

[30] S. Ryu and T. Takayanagi, "Holographic derivation of entanglement entropy from AdS/CFT," Phys. Rev. Lett. 96, 181602 (2006) [hep-th/0603001].

[31] T. Numasawa, N. Shiba, T. Takayanagi and K. Watanabe, "EPR Pairs, Local Projections and Quantum Teleportation in Holography," JHEP 1608, 077 (2016) [arXiv:1604.01772 [hep-th]].

[32] F. Dominguez, C. Marquet, A. H. Mueller, B. Wu and B. W. Xiao, Nucl. Phys. A 811, 197 (2008) [arXiv:0803.3234 [nucl-th]]. 